\title{
Real-space quantum-based refinement for cryo-EM: Q|R\#3
}

Lum Wang1, Holger Kruse ${ }^{2}$, Oleg V. Sobolev ${ }^{3}$ Nigel W. Moriarty ${ }^{3}$, Mark P. Waller ${ }^{4 *}$, Pavel V. Afonine ${ }^{3 *}$ and Malgorzata Biczysko ${ }^{1 *}$

${ }^{1}$ International Center for Quantum and Molecular Structures, Shanghai University, Shanghai 200444, People's Republic of China

2Institute of Biophysics of the Czech Academy of Sciences, Brno, Czech Republic

${ }^{3}$ Molecular Biosciences and Integrated Bioimaging, Lawrence Berkeley National Laboratory, Berkeley, CA 94720, USA

${ }^{4}$ Pending AI Pty Ltd., iAccelerate, Innovation Campus, North Wollongong, 2500, Australia.

*e-mail: PAfonine@lbl.gov, mark@pending.ai, biczysko@shu.edu.cn

Keywords: quantum refinement, protein, crystallography, cryo-EM, real-space refinement, CompaRama

\section{Synopsis}

The implementation of quantum-based real-space refinement in qr.refine is described.

\begin{abstract}
Electron cryo-microscopy (cryo-EM) is fast becoming a major competitor to X-ray crystallography especially for large structures that are difficult or impossible to crystallize. While recent spectacular technology improvements are leading to significantly higher resolution of three-dimensional reconstructions, the average quality of cryo-EM maps is still on the low-resolution end of the range compared to crystallography. A long-standing challenge for atomic model refinement has been the production of stereochemically meaningful models for this resolution regime. Here we demonstrate how including accurate model geometry restraints derived from $a b$ initio quantum-chemical calculations (HF-D3/6-31G) can improve the refinements of an example structure (chain A of $3 \mathrm{j} 63$ ). The robustness of the procedure is tested for additional structures with up to $7 \mathrm{k}$ atoms (3a5x, and chain $\mathrm{C}$ of $5 \mathrm{fn} 5$ ) by means of the less expensive semi-empirical (GFN1-xTB) model.
\end{abstract}


Necessary algorithms enabling real-space quantum refinement are implemented in the latest version of qr.refine and are described herein.

\section{Introduction}

Improvements in cryo-EM technology resulted in a rapidly increasing number of three-dimensional reconstructions of $4.5 \AA$ and better (Kühlbrandt, 2014, Henderson, 2015, Nogales, 2015, Orlov et al., 2017, Baldwin et al., 2018) allowing interpretation of corresponding maps in terms of atomic models. This prompted the active development of map improvement (Terwilliger, Sobolev, et al., 2018; Terwilliger, Ludtke, et al., 2019), model building (Terwilliger, Adams, et al., 2018, 2019), refinement (Afonine, Poon, et al., 2018) and validation methods (Afonine, Klaholz, et al., 2018). It is a common knowledge that atomic model refinement can be challenging at resolutions worse than about 3-3.5 $\AA$ (Headd et al., 2012). This is because beyond this resolution the amount of detail in the experimental data is insufficient to resolve structural features of proteins such as secondary-structure or rotameric states of amino-acid residues at an atomic level. This in turn requires extra information to be used in refinement, for example: Ramachandran plot, rotamer, secondary-structure or reference model restraints (for a summary, see for example Afonine, Poon, et al., 2018). Quantum-mechanical (QM) calculations generate model geometries $a b$ initio and thus do not rely on empirical libraries, such as the commonly used Monomer Library (Vagin \& Murshudov, 2004; Vagin et al., 2004) nor any other ad hoc restraints. Historically, quantum refinements are associated with either impractical runtimes even for small proteins or methods that focus on QM calculations only for the macromolecular region of interest, such as a ligand-binding pocket or an enzyme's active site, while treating the rest of the molecule with a classical approach (Ryde, 2003; Ryde \& Nilsson, 2003b, a; Nilsson et al., 2004; Borbulevych et al., 2014). Recently we have demonstrated that the entire atomic model can benefit from a full QM treatment (Zheng, Reimers, et al., 2017) being the first to enable full protein crystal structure refinement using QM based geometry gradients in place of the gradients from classical restraints (Zheng, Moriarty, et al., 2017).

Quantum Refinement (Q $\mid \mathrm{R}$; www.qrefine.com) is an international collaborative project with the goal to create easy to use software for full structure refinement using gradients derived from quantum-based calculations. $Q \mid R$ is built upon the CCTBX library (Grosse-Kunstleve et al., 2002) and operates as a plugin to the Phenix software (Liebschner et al., 2019). It uses Atomic Simulation Environment (ASE) adaptors (Bahn et al., 2002) to interface to a number of available QM packages such 
as TeraChem (Ufimtsev et al., 2009; Titov et al., 2012), Orca (Neese, 2012), xtb (Grimme et al., 2017; Bannwarth et al., 2019), MOPAC (Stewart, 2016), TURBOMOLE (Furche et al., 2014), Gaussian (Frisch et al., 2016) and more. Q|R is a work in progress with set challenges that we address as the project progresses. Describing fundamentals (Zheng, Reimers, et al., 2017), proving concept (Q|R\#1, Zheng, Moriarty, et al., 2017), handling crystallographic symmetry (Q|R\#2, Zheng et al., 2020), supporting cryo-EM (Q|R\#3, this manuscript), alternative conformations $(\mathrm{Q} \mid \mathrm{R} \# 4$, manuscript in preparation) and benchmarking different QM methods to optimize speed and accuracy of calculations, are examples of such challenges and milestones.

Recent development of real-space methods and tools in CCTBX enabled the implementation of classic real-space refinement in Phenix (Afonine, Poon, et al., 2018) specifically designed for cryo-EM but also supports crystallographic data. Since Q|R is built on CCTBX and is tightly integrated with Phenix, it was possible to re-use the key components of CCTBX to implement quantum-based real-space refinement in Q|R. Herein we describe the implementation details and demonstrate the utility of the method and reliability of geometry restraints derived from ab initio quantum-chemical calculations using select examples.

\section{Methods}

\subsection{Real-space refinement in $Q / R$}

Similarly to reciprocal-space refinement (Afonine et al., 2012), the refinement target function is a composite of two terms

$T=w T_{\text {data }}+T_{\text {restraints }}$

where $T_{\text {data }}$ scores the model-to-data fit, $T_{\text {restraints }}$ represents a priori information about the problem and $w$ is an empirical weight factor that allows to maximize model-to-data fitting while enforcing the model to conform to the prior knowledge. Except for rare cases when ultra-high resolution $\left(0.7 \AA\right.$ or better $\left.{ }^{1}\right)$ data are available (Dauter et al., 1992; Bönisch et al., 2005; Wang et al., 2007; Hirano et al., 2016), refinement of macromolecular models always requires using $T_{\text {restraints }}$ to compensate for the limited quality of experimental data (e.g. finite resolution).

${ }^{1}$ Different sources quote different threshold for defining ultra-high resolution on seemingly arbitrary grounds. Here we use $0.7 \AA$ as described by Urzhumtsev et al. (2009) because they seem to provide most solid rationale for the choice of this resolution cutoff. 
In contrast to reciprocal-space refinement, the experimental data used in (1) are not structure factor amplitudes or intensities but a real-space map. This allows the calculations to be done locally, which in turn enables faster calculations and larger refinement convergence radii (Afonine, Poon, et al., 2018). Indeed, in reciprocal space refinement all atoms and all $h k l$ Miller indices corresponding to measured data are required in order to match model-calculated structure factors with measured ones. In real-space refinement the three-dimensional reconstruction, the map, represents the experimental data and atoms can move locally to optimize fit to the map. This, for example, allows use of only a fraction of the atomic model to find the optimal weight $w$ in (1), which is computationally efficient compared to using the whole model as implemented in phenix.real_space_refine and described by Afonine, Poon, et al. (2018).

$\mathrm{Q} \mid \mathrm{R}$ uses the cctbx.iotbx module of CCTBX to import an MRC formatted map (Winn et al., 2011) and atomic model in PDB (Bernstein et al., 1977) or mmCIF formats (Adams et al., 2019). The cctbx.maptbx module is used to perform calculation of (1) and its gradients as described in Afonine, Poon, et al. (2018). The cctbx.maptbx.real_space_refinement_simple module is used to carry out the optimization of (1). In this work, it is assumed that a cryo-EM map is sampled in a P1 symmetry box with the model situated in its center and, unlike for crystallography, no periodicity needs to be taken into account. Optimization of the weight $w$ in (1) uses the same idea as in phenix.real_space_refine but is implemented differently owing to specifics of QM calculations. phenix.real_space_refine systematically probes the weight in the range from very small (least emphasis on the data) to very large (most emphasis on the data). This can cause problems in qr.refine because a trial refinement run with a very large weight is likely to yield a very geometrically distorted atomic model that in turn is likely to impede QM calculations. Therefore in quantum refinement the order of trial weights is important. Here the weights are sampled from smallest to largest and for each trial weight several parameters are monitored. These are, the root-mean-squared deviation (RMSD) of bond lengths from "ideal" library values (Vagin \& Murshudov, 2004; Vagin et al., 2004) as in the phenix.real_space_refine, and additionally the percentage of residues in the favored region of the Ramachandran plot (\% favored), percentage of rotamer outliers, $\mathrm{C} \beta$ deviation and the Clash-score. These define the overall quality of the model with deviation growing as the trial weight increases; the weight search terminates once the bond RMSD reaches the predefined threshold or any other parameter worsen. The refinement was performed with the qr.refine version v1.0-176 coupled to the Phenix 1.17. 


\subsection{Test model selection, preparation, refinement setup and analysis}

Three atomic models and corresponding maps have been selected from the Protein Data Bank (wwPDB consortium, 2019) and Electron Microscopy Data Bank (Lawson et al., 2011) with a resolution range between 3.8 and $4.3 \AA$ (Table 1). The 3a5x model was used as is, while only chains A and $C$ were used from $3 \mathrm{j} 63$ and $5 \mathrm{fn} 5$, respectively. Each model was subject to a fully automated preparation for quantum refinement using the qr.finalise tool (Zheng, Moriarty, et al., 2017) of the Q|R package; this includes completing missing side chains and adding all theoretically possible (but missing) hydrogen atoms. For partial models ( $3 \mathrm{j} 63$ and $5 \mathrm{fn} 5$ ) a box with the map around corresponding chains was extracted using the phenix.map_box tool (Liebschner et al., 2019).

All refinements were performed using qr.refine. Quantum refinement was performed using the TeraChem (Ufimtsev \& Martínez, 2009; Titov et al., 2012) and xtb (Grimme et al., 2017; Bannwarth et al., 2019) packages as QM engines. Classic refinement (also referred here to as CCTBX refinement) used geometry restraints as implemented in CCTBX and used in Phenix refinement programs such as phenix.refine (Afonine et al., 2012) and phenix.real_space_refine (Afonine, Poon, et al., 2018). Classic refinement used only a standard set of geometry restraints: restraints on covalent bond lengths and bond angles, dihedral angles, chiralities, planarities and non-bonded repulsion (Engh \& Huber, 1991, Grosse-Kunstleve et al., 2002, Afonine et al., 2004; Moriarty et al., 2016). TeraChem refinement was performed using the Hartree-Fock (Slater, 1951) method with Grimme's dispersion correction D3 (Grimme et al., 2010) in conjunction with the 6-31G basis set (Hehre et al., 1972) accounting for the polar (water, $\varepsilon=78$ ) environment by means of the COSMO polarizable continuum model (Liu et al, 2015, Barone \& Cossi, 1998, Truong \& Stefanovich, 1995). In what follows we will refer to this method as HF-D3/6-31G or simply TeraChem (TC). xtb refinement was performed with the original GFN-xTB model (Grimme et al., 2017), nowadays denoted GFN1-xTB, and including the solvent effect by means of xtb's internal generalized Born solvation model. Atomic model fragmentation is the key step in the refinement procedure that makes QM calculations practical even for large molecules. Fragmentation as described in Zheng, Moriarty, et al. (2017) was used in all refinement runs. We refer to model geometry optimization (model regularization) as a special refinement regime using a modified version of (1) with the $T_{\text {data }}$ term entirely omitted.

Standard geometric criteria (Morris et al., 1992) along with MolProbity (Williams et al., 2018) tools were used to assess the quality of the atomic models before and after 
refinement. The distribution of the protein $(\varphi, \psi)$ angles is indicative of refinement success and generally is sensitive to the model parameterization used (e.g., restraints). Thus, assessing Ramachandran plots is an integral part of model validation. However, comparison of two or more Ramachandran plots is not straightforward because dots on the plot that represent $(\varphi, \psi)$ pairs are not labeled and for large models overlap often appearing as a single blob. Following Kleywegt \& Jones (1996) we have implemented the phenix.comparama tool in Phenix that allows the tracking of changes of $(\varphi, \psi)$ angles on the Ramachandran plot. The tool takes two models, for example, before and after refinement, and makes a Ramachandran plot for the latter model. Then for each $(\varphi, \psi)$ pair that has moved from one region of the plot to another it draws an arrow that begins at the position of this pair in the starting model. Light green arrows show residues that left the outlier region, red arrows are for residues that became outliers, orange indicate favored-to-allowed transitions, and green stands for allowed-to-favored transitions.

\section{Results and discussion}

Table 1 summarizes data, model and model-to-data fit statistics for initial models and maps as obtained from $\mathrm{PDB} / \mathrm{EMDB}$ along with the results of the refinement runs: quantum (TeraChem and/or xtb) and classic with two different ways to define the weight in (1). We observe that quantum refinement and optimization (see below, and also (Zheng et al., 2020, Zheng, Reimers, et al., 2017, Zheng, Moriarty, et al., 2017) consistently produce models with RMSDs from library values for covalent bonds and angles of around $0.015 \AA$ and 1.5 degrees, respectively, matching the community consensus on the targets for these metrics (see discussion and set of references in Afonine, P. V., Klaholz et al., 2018). At the same time low-resolution experimental data cannot justify these rather large deviations in these values, likely resulting in fitting the model to noise. This suggests that for low-resolution refinements the choice of weight in (1) should be such that the refinement produces models with bond and angle RMSDs as small as achievable in pure geometry regularization. With this in mind we performed two independent runs of classic refinement: one using the weight in (1) that results in bond and angle RMSDs of approximately $0.015 \AA$ and 1.5 degrees (referred to as CCTBX loose in Table 1), and the other one that produces models with nearly idealized covalent geometry (referred to as CCTBX tight in Table 1).

Firstly, we note that all three initial models have rather poor geometry. Indeed, according to MolProbity (Chen et al., 2010) a geometrically sound model is expected to have $98 \%$ or more residues in the favored region of the Ramachandran plot and less than $0.05 \%$ in disallowed, less than $0.3 \%$ of residue side chains being rotamer 
outliers, no $\mathrm{C}_{\beta}$ deviations and a low clashscore (the lower the better, typically 10-15 or below). None of the three models pass these criteria. The Ramachandran Z-score (Rama-Z) (Hooft et al., 1997, van Beusekom et al., 2018, Sobolev et al. 2020) provides a convenient numeric estimate of the protein main-chain conformation validity. The rule-of-thumb interpretation of the Ramachandran plot Z-score (Sobolev et al., 2020) can be summarized as $\mid$ Rama-Z $\mid>3$ is poor indicating improbable backbone geometry, $2<\mid$ Rama-Z $\mid<3$ unlikely yet possible, and |Rama$\mathrm{Z} \mid<2$ is good, normal backbone. Only one model, $5 \mathrm{fn} 5$, has this metric within the good range (0.8). However, considering the Rama- $Z$ score separately for different secondary structure classes (such as helices, sheets and loops), we find that the Rama-Z score for helices is 1.6 (good) while it is -4.2 for loops (poor), with the standard deviations of 0.4 and 0.8 , respectively. This means that overall the model main-chain conformation is problematic.

Figure 1 shows the Ramachandran plots for the deposited 3j63 as well as for rerefined models. As already stated, the deposited model (Fig. 1 a) is poor in both the Rama-Z score (-4.5) and percentage of residues in favored region of the plot (85.4). The TeraChem calculations transform these values to the "good" Rama-Z range (1.3) and perfect percentage in favored region (Fig $1 \mathrm{~b}, \mathrm{c}$ ). The xtb model is also an improvement $(-2.7,98.9)$ on the initial model moving both statistics to the edge or into "good" regions in each case. The better quality of the TeraChem refinement comes with a multi-fold increased computational cost (from about $14 \mathrm{~h}$ for xtb on a 64-core CPU to $150 \mathrm{~h}$ for TeraChem on 8 nodes with 4 GPUs each).

Classic real-space refinement performs drastically different depending on the choice of the weight scale in (1). Relaxing geometry restraints (CCTBX loose) to produce comparable bond and angle deviations to quantum refinement results in models with very poor metrics such as Ramachandran plot (Fig. 1f), Rama-Z score, rotamers and clashscore (Table 1). Using very tight restraints (CCTBX tight) yields models with nearly idealized stereochemistry, while having unlikely secondary-structure geometry parameters as judged by the Ramachandran plot (Fig. 1e) and the Rama-Z score (Table 1).

Extending the $\mathrm{xtb}$ and classical refinements to include $3 \mathrm{a} 5 \mathrm{x}$ and $5 \mathrm{fn} 5$ provides better statistics. The results covering the three models are shown in figure 2 . The Rama-Z values for the $x t b$ and classical refinements are similar to the single $3 \mathrm{j} 63$ model. Furthermore, for the xtb the distribution of residues on the Ramachandran plot is notably better as it follows the most probable regions in both alpha and beta segments of the plot (Fig. 2b). All the geometry parameter improvements occur at no expense to the model to data (map) fit, as indicated by the $\mathrm{CC}_{\text {mask }}$ values (Afonine 
et al., 2019) shown in table 1 . Moreover, for $3 a 5 x$ and 5 fn 5 quantum refinement significantly reduced the number of residue side-chain rotamer outliers compared to starting models. Fixing a rotamer outlier often requires traversing energy barriers in $\chi$-angle space, which is unachievable by well-behaved gradient-driven optimization requiring additional methods such as simulated annealing (Brunger \& Rice, 1997) or torsion exhaustive searches in $\chi$-angle space (Oldfield, 2001). This explains the fact that none of the refinements produced outlier-free models. Also, we note that in some cases quantum refinement produced models with an elevated number of rotamer outliers compared to the CCTBX tight refinements. The reason for this is three-fold: 1) tight CCTBX restraints result in very strong nonbonded repulsions that force atoms into more favorable (but not necessarily most correct) conformations, 2) map quality in all three cases does not allow unambiguous placement of side-chains and as a result 3) allows hydrogen bond interactions between side-chain and neighbor atoms (often spurious due to poor initial model quality) that can generate sufficient strain forcing the side chain into a rotamer outlier.

Expectedly, all classic refinements using loose restraints (CCTBX loose, Table 1) produce noticeably higher (better) map-model correlations $\mathrm{CC}_{\text {mask }}$ while also all of them show poorer model geometry. This can be interpreted as over-fitting of the data. We also note that classic refinements with tight restraints produce models with very similar $\mathrm{CC}_{\text {mask }}$ compared to $\mathrm{xtb}$ or $\mathrm{TC}$. This means that these geometric improvements are on the scale of magnitude that can hardly be validated by the experimental data.

As follows from Table 1, the most notable improvement to the model resulting from quantum refinement was the quality of the protein main chain conformations. This is evidenced by significantly improved Ramachandran plot statistics, with significantly better Rama-Z scores obtained with TeraChem. The data resolution in all three selected examples is far from atomic and thus cannot validate this improvement. This led to the idea of performing an additional test that shows the superiority of QM restraints, and compares directly results from TeraChem and xtb. For this test we have selected one of the best-quality available model from the PDB solved at ultra-high resolution of $0.66 \AA$ (1us0; Fig. 3a). For simplicity, we left out all non-protein atoms and atoms in alternative conformations (keeping just one conformer with the highest occupancy and resetting its occupancy to unity). Also, we created another copy of this model with perturbations of the atomic coordinates (Fig. 3b). We then subjected these two models to the pure geometry optimization using the TeraChem (HF-D3/6-31G), xtb (GFN1-xTB) packages and CCTBX, six optimizations were run in total. We rationalize this test as following. Given the high- 
resolution data used to determine the 1 us 0 model, we can assume that this model accurately represents the true structure. In turn, we expect that a better potential used to optimize the original model should not move or perturb it in any significant way, while the optimized perturbed model is expected to move back closer to the unperturbed original model. Indeed, Figure 3 shows that TeraChem based optimization brought the perturbed model very close to the original one (Fig. 3d) while not moving the original model significantly (Fig. 3c). The xtb approach yields some small deviations to the original model (Fig. 3e), but significantly improved the perturbed model (Fig. 3f). In contrast, optimization using classic restraints moved the perturbed model further away (Fig. 3h) from the original one. Moreover, the classic optimization substantially perturbed the original model (Fig. 3g). This test shows that replacement of classic restraints by the QM restraints result in more realistic molecular models even in the absence of experimental data.

\section{Conclusions}

Here we presented the new capability of the Q|R software package to perform realspace refinement enabling quantum refinement of cryo-EM derived models. The only input information required to run the refinement are the atomic model and the map. The atomic model is expected to be well-refined using standard classic refinement and be atom-complete. We once again demonstrated the superiority of the quantum-based restraints over the classic approach. In the three examples considered, we observe significant improvement of model geometry metrics after quantum refinement compared to the original models and to models after classic refinements. The HF-D3/6-31G level of theory performs better than the GFN1-xTB model for our refinements. A new tool for graphical comparison of Ramachandran plots (phenix.comparama) has been developed to aid this work and is now part of Phenix software suite.

Using quantum refinement from $Q \mid R$ project requires installing $Q \mid R$ software (www.qrefine.com), Phenix (www.phenix-online.org) and one of the supported quantum refinement programs, such as TeraChem, Orca, or xtb.

Re-refinement results and related data are available at: https://github.com/qrefine/QR3-RSR-cryo-EM.

\section{Acknowledgments}


This work was enabled by the financial support from the National Natural Science Foundation of China (Grant No. 31870738) and support from the Shanghai Eastern Scholar Program. HK acknowledges support by the ERDF project SYMBIT (CZ.02.1.01/0.0/0.0/15_003/0000477).

\section{Literature}

Adams, P.D., Afonine, P.V., et al. (2019). Acta Crystallogr D Struct Biol. 2019 Apr 1; 75(Pt 4): 451-454.

Afonine, P. \& Urzhumtsev, A. (2004). Acta Crystallographica Section A: Foundations of Crystallography 60, 19-32.

Afonine, P. V., Grosse-Kunstleve, R. W., Echols, N., Headd, J. J., Moriarty, N. W., Mustyakimov, M., Terwilliger, T. C., Urzhumtsev, A., Zwart, P. H. \& Adams, P. D. (2012). Acta Crystallographica Section D: Biological Crystallography 68, 352-367.

Afonine, P. V., Klaholz, B. P., Moriarty, N. W., Poon, B. K., Sobolev, O. V., Terwilliger, T. C., Adams, P. D. \& Urzhumtsev, A. (2018). Acta Crystallogr D Struct Biol 74, 814-840.

Afonine, P. V., Lunin, V. Y., Muzet, N. \& Urzhumtsev, A. (2004). Acta Crystallographica Section D: Biological Crystallography 60, 260-274.

Afonine, P. V., Poon, B. K., Read, R. J., Sobolev, O. V., Terwilliger, T. C., Urzhumtsev, A. \& Adams, P. D. (2018). Acta Crystallographica Section D: Structural Biology 74.

Bahn, S. R., Jacobsen, K. W. \& Engineering (2002). Computing in Science 4, 56-66.

Baldwin, P. R., Tan, Y. Z., Eng, E. T., Rice, W. J., Noble, A. J., Negro, C. J., Cianfrocco, M. A., Potter, C. S. \& Carragher, B. (2018). Current opinion in microbiology 43, 18.

Bannwarth, C., Ehlert, S. \& Grimme, S. (2019). Journal of chemical theory computation 15, 1652-1671.

Barone, V. \& Cossi, M. (1998). The Journal of Physical Chemistry A 102, 1995-2001.

Bernstein, F. C., Koetzle, T. F., Williams, G. J., Jr, M. E., Brice, M. D., Rodgers, J. R., Kennard, O., Shimanouchi, T. \& Tasumi, M. (1977). European Journal of Biochemistry 185, 584-591.

Bönisch, H, Schmidt, C.L., Bianco, P. \& Ladenstein, R. (2005). Acta Crystallogr D Biol Crystallogr. Jul; 61(Pt 7): 990-1004.

Borbulevych, O. Y., Plumley, J. A., Martin, R. I., Merz, K. M. \& Westerhoff, L. M. (2014). Acta Crystallographica Section D: Biological Crystallography 70, 1233-1247.

Brünger, A.T. \& Rice, L.M. (1997). Methods Enzymol. 277. 243-269.

Chen, V. B., Arendall, W. B., Headd, J. J., Keedy, D. A., Immormino, R. M., Kapral, G. J., Murray, L. W., Richardson, J. S. \& Richardson, D. C. (2010). Acta Crystallographica Section D: Biological Crystallography 66, 12-21.

Dauter, Z., L. C. Sieker \& K. S. Wilson. (1992). Acta Crystallogr B. 1992 Feb 1; 48(Pt 1): 42-59. 
Engh, R. A. \& Huber, R. (1991). Acta Crystallographica Section A: Foundations of Crystallography 47, 392-400.

Frisch, M., Trucks, G., Schlegel, H. B., Scuseria, G., Robb, M., Cheeseman, J., Scalmani, G., Barone, V., Petersson, G. et al., (2016). Inc., Wallingford, CT 2016.

Furche, F., Ahlrichs, R., Hättig, C., Klopper, W., Sierka, M. \& Weigend, F. (2014). Wiley Interdisciplinary Reviews: Computational Molecular Science 4, 91-100.

Grimme, S., Antony, J., Ehrlich, S. \& Krieg, H. (2010). J Chem Phys 132, 154104.

Grimme, S., Bannwarth, C. \& Shushkov, P. (2017). Journal of chemical theory computation 13, 1989-2009.

Grosse-Kunstleve, R. W., Sauter, N. K., Moriarty, N. W. \& Adams, P. D. (2002). Acta Crystallographica Section D: Biological Crystallography 35, 126-136.

Hirano, Y., Takeda, K. \& Miki, K. (2016). Nature 534, 281-284.

Headd, J. J., Echols, N., Afonine, P. V., Grosse-Kunstleve, R. W., Chen, V. B., Moriarty, N. W., Richardson, D. C., Richardson, J. S. \& Adams, P. D. (2012). Acta Crystallographica Section D: Biological Crystallography 68, 381-390.

Hehre, W. J., Ditchfield, R. \& Pople, J. A. (1972). The Journal of Chemical Physics 56, 2257-2261.

Henderson, R. (2015). Archives of biochemistry biophysics 581, 19-24.

Hooft, R. W., Sander, C. \& Vriend, G. (1997). Bioinformatics 13, 425-430.

Kleywegt, G. J. \& Jones, T. A. (1996). Structure 4, 1395-1400.

Kühlbrandt, W. (2014). Science 343, 1443-1444.

Lawson, C. L., Baker, M. L., Best, C., et al. (2011). 39(Database issue), D456-D464.

Liebschner, D., Afonine, P. V., Baker, M. L., Bunkóczi, G., Chen, V. B., Croll, T. I., Hintze, B., Hung, L.-W., Jain, S. \& McCoy, A. J. (2019). Acta Crystallographica Section D: Structural Biology 75, 861-877.

Liu, F., Luehr, N, Kulik, J. K., Martinez, T. J. (2015). J. Chem. Theory Comput. 11, 31313144

Moriarty, N. W., Tronrud, D. E., Adams, P. D., \& Karplus, P. A. (2016). Acta crystallographica. Section D, Structural biology, 72(Pt 1), 176-179.

Morris, A. L., MacArthur, M. W., Hutchinson, E. G. \& Thornton, J. M. (1992). Proteins: Structure, Function, Bioinformatics 12, 345-364.

Neese, F. (2012). Wiley Interdisciplinary Reviews: Computational Molecular Science 2, 73-78.

Nilsson, K., Hersleth, H.-P., Rod, T. H., Andersson, K. K. \& Ryde, U. (2004). Biophysical journal 87, 3437-3447.

Nogales, E. (2015). Nature methods 13, 24.

Oldfield, T.J. (2001). Acta Crystallogr D Biol Crystallogr. 57(Pt 1): 82-94.

Orlov, I., Myasnikov, A. G., Andronov, L., Natchiar, S. K., Khatter, H., Beinsteiner, B., Ménétret, J. F., Hazemann, I., Mohideen, K. \& Tazibt, K. (2017). Biology of the Cell 109, 81-93.

Ryde, U. (2003). Current opinion in chemical biology 7, 136-142.

Ryde, U. \& Nilsson, K. (2003a). J Journal of the American Chemical Society 125, 14232-14233.

Ryde, U. \& Nilsson, K. (2003b). Journal of Molecular Structure: THEOCHEM 632, 259275.

Slater, J. C. (1951). Physical review 81, 385. 
Sobolev, O. V., Afonine, P. V., Moriarty, N. W., Hekkelman, M. L., Joosten, R. P., Perrakis, A., Adams,P. D., bioRxiv DOI: 10.1101/2020.03.26.010587

Stewart, J. J. (2016). Stewart Computational Chemistry: Colorado Springs, CO, USA, .

Tagari, M., Newman, R., Chagoyen, M., Carazo, J.-M. \& Henrick, K. (2002). Trends in biochemical sciences 27, 589-589.

Terwilliger, T. C., Adams, P. D., Afonine, P. V. \& Sobolev, O. V. (2018). Nature methods $15,905$.

Terwilliger, T. C., Adams, P. D., Afonine, P. V. \& Sobolev, O. V. (2019). Protein Science.

Terwilliger, T. C., Ludtke, S. J., Read, R. J., Adams, P. D. \& Afonine, P. V. (2019). BioRxiv, 845032.

Terwilliger, T. C., Sobolev, O. V., Afonine, P. V. \& Adams, P. D. (2018). Acta Crystallographica Section D: Structural Biology 74.

Titov, A. V., Ufimtsev, I. S., Luehr, N., Martinez, T. J. \& computation (2012). Journal of chemical theory $\mathbf{9}, 213-221$.

Truong, T. N. \& Stefanovich, E. V. (1995). Chemical Physics Letters 240, 253-260.

Ufimtsev, I. S., Martinez, T. J. \& Computation (2009). Journal of Chemical Theory 5, 2619-2628.

Urzhumtsev, A., Afonine, P. V., \& Adams, P. D. (2009). On the use of logarithmic scales for analysis of diffraction data. Acta crystallographica. Section D, Biological crystallography, 65(Pt 12), 1283-1291.

Vagin, A. A. \& Murshudov, G. N. (2004). IUCr Comput. Comm. Newsl. 4, 59-72.

Vagin, A. A., Steiner, R. A., Lebedev, A. A., Potterton, L., McNicholas, S., Long, F. \& Murshudov, G. N. (2004). Acta Crystallographica Section D: Biological Crystallography 60, 2184-2195.

van Beusekom, B., Joosten, K., Hekkelman, M. L., Joosten, R. P. \& Perrakis, A. (2018). IUCrJ 5.

Wang, J., Dauter, M., Alkire, R., Joachimiak, A. \& Dauter, Z. (2007). Acta Crystallogr D Biol Crystallogr. Dec; 63(Pt 12): 1254-1268.

Winn, M. D., Ballard, C. C., Cowtan, K. D., Dodson, E. J., Emsley, P., Evans, P. R., Keegan, R. M., Krissinel, E. B., Leslie, A. G. \& McCoy, A. (2011). Acta Crystallographica Section D: Biological Crystallography 67, 235-242.

Williams, C.J., Hintze, B.J., Headd, J.J., Moriarty, N.W., Chen, V.B., Jain, S., Prisant, M.G., Lewis, S.M., Videau, L.L., Keedy, D.A., Deis, L.N., Arendall, W.B. III, Verma, V., Snoeyink, J.S., Adams, P.D., Lovell, S.C., Richardson, J.S., Richardson D.C. (2018) ProtSci 27 293-315.

wwPDB consortium. (2019). Nucleic Acids Research, Volume 47, Issue D1, 08 January 2019, Pages D520-D528.

Zheng, M., Biczysko, M., Xu, Y., Moriarty, N. W., Kruse, H., Urzhumtsev, A., Waller, M. P. \& Afonine, P. V. (2020). Acta Crystallographica Section D: Structural Biology 76, 41-50.

Zheng, M., Moriarty, N. W., Xu, Y., Reimers, J. R., Afonine, P. V. \& Waller, M. P. (2017). Acta Crystallographica Section D: Structural Biology 73, 1020-1028.

Zheng, M., Reimers, J. R., Waller, M. P. \& Afonine, P. V. (2017). Acta Crystallographica Section D: Structural Biology 73, 45-52. 
bioRxiv preprint doi: https://doi.org/10.1101/2020.05.25.115386; this version posted May 28, 2020. The copyright holder for this preprint (which was not certified by peer review) is the author/funder. All rights reserved. No reuse allowed without permission. 


\begin{tabular}{|c|c|c|c|c|c|c|c|c|c|c|c|c|c|c|}
\hline \multicolumn{2}{|c|}{ PDB/EMDB } & & \multicolumn{4}{|c|}{ 3j63/5830 } & \multicolumn{4}{|c|}{$5 \mathrm{fn} 5 / 3240$} & \multicolumn{4}{|c|}{$3 a 5 x / 1641$} \\
\hline \multicolumn{2}{|c|}{ Atoms/residues } & & \multicolumn{4}{|c|}{$1436 / 91$} & \multicolumn{4}{|c|}{$3787 / 243$} & \multicolumn{4}{|c|}{$7194 / 494$} \\
\hline \multicolumn{2}{|c|}{ Resolution $(\AA ̊)$} & & \multicolumn{4}{|c|}{3.8} & \multicolumn{4}{|c|}{4.3} & \multicolumn{4}{|c|}{4} \\
\hline Metric & Model & Initial & $\begin{array}{l}\text { CCTBX } \\
\text { (loose) }\end{array}$ & $\begin{array}{l}\text { CCTBX } \\
\text { (tight) }\end{array}$ & xtb & $\mathrm{TC}$ & Initial & $\begin{array}{l}\text { CCTBX } \\
\text { (loose) }\end{array}$ & $\begin{array}{l}\text { CCTBX } \\
\text { (tight) }\end{array}$ & $\mathrm{xtb}$ & Initial & $\begin{array}{l}\text { ССТВX } \\
\text { (loose) }\end{array}$ & $\begin{array}{l}\text { CСTBX } \\
\text { (tight) }\end{array}$ & $\mathrm{xtb}$ \\
\hline \multicolumn{2}{|c|}{$\mathrm{CC}_{\text {mask }}$} & 0.67 & 0.74 & 0.67 & 0.61 & 0.67 & 0.64 & 0.72 & 0.66 & 0.64 & 0.31 & 0.55 & 0.32 & 0.33 \\
\hline \multirow{2}{*}{ RMSD } & Bonds $(\AA ̊)$ & 0.004 & 0.015 & 0.002 & 0.012 & 0.015 & 0.012 & 0.015 & 0.002 & 0.009 & 0.014 & 0.015 & 0.001 & 0.010 \\
\hline & Angles $\left({ }^{\circ}\right)$ & 1.22 & 1.30 & 0.48 & 1.82 & 1.61 & 2.00 & 1.40 & 0.50 & 1.57 & 1.65 & 1.62 & 0.39 & 1.82 \\
\hline \multirow{3}{*}{$\begin{array}{c}\text { Rama. } \\
\text { plot } \\
(\%)\end{array}$} & Favored & 85.4 & 55.1 & 96.6 & 98.9 & 100 & 90.9 & 61.0 & 89.2 & 94.6 & 86.6 & 57.1 & 92.5 & 92.7 \\
\hline & Outliers & 4.5 & 16.9 & 0 & 0 & 0 & 2.1 & 12.0 & 0.8 & 0.8 & 2.9 & 19.5 & 0.4 & 0.8 \\
\hline & Rama-Z & -4.5 & -8.1 & -3.5 & -2.7 & -1.3 & 0.8 & -7.3 & -3.3 & -2.3 & -4.4 & -7.4 & -3.5 & -2.9 \\
\hline \multicolumn{2}{|c|}{ Rotamer outliers (\%) } & 5.5 & 19.2 & 5.5 & 6.9 & 4.1 & 18.1 & 26.4 & 7.3 & 7.3 & 8.7 & 27.4 & 2.1 & 3.3 \\
\hline \multicolumn{2}{|c|}{ Clashscore } & 50.8 & 43.9 & 3.5 & 1.4 & 1.4 & 14.3 & 69.8 & 6.3 & 1.9 & 17.8 & 89.1 & 2.4 & 2.8 \\
\hline \multicolumn{2}{|c|}{$\mathrm{C} \beta$ deviation } & 0 & 0 & 0 & 0 & 0 & 2.3 & 0 & 0 & 0 & 0 & 0 & 0 & 0 \\
\hline
\end{tabular}

Table 1. Summary of data, model and model to data fit statistics for initial model (Initial) and models after refinement using CCTBX (with tight and loose geometry restraints) and quantum restraints derived from TeraChem (TC) or GFN1-xTB (xtb). $\mathrm{CC}_{\text {mask }}$ reports the correlation between the model-generated and experimental maps. Atom/residue counts for $5 \mathrm{nf5}$ and $3 \mathrm{a} 5 \mathrm{x}$ are shown for partial models actually used in refinements. 

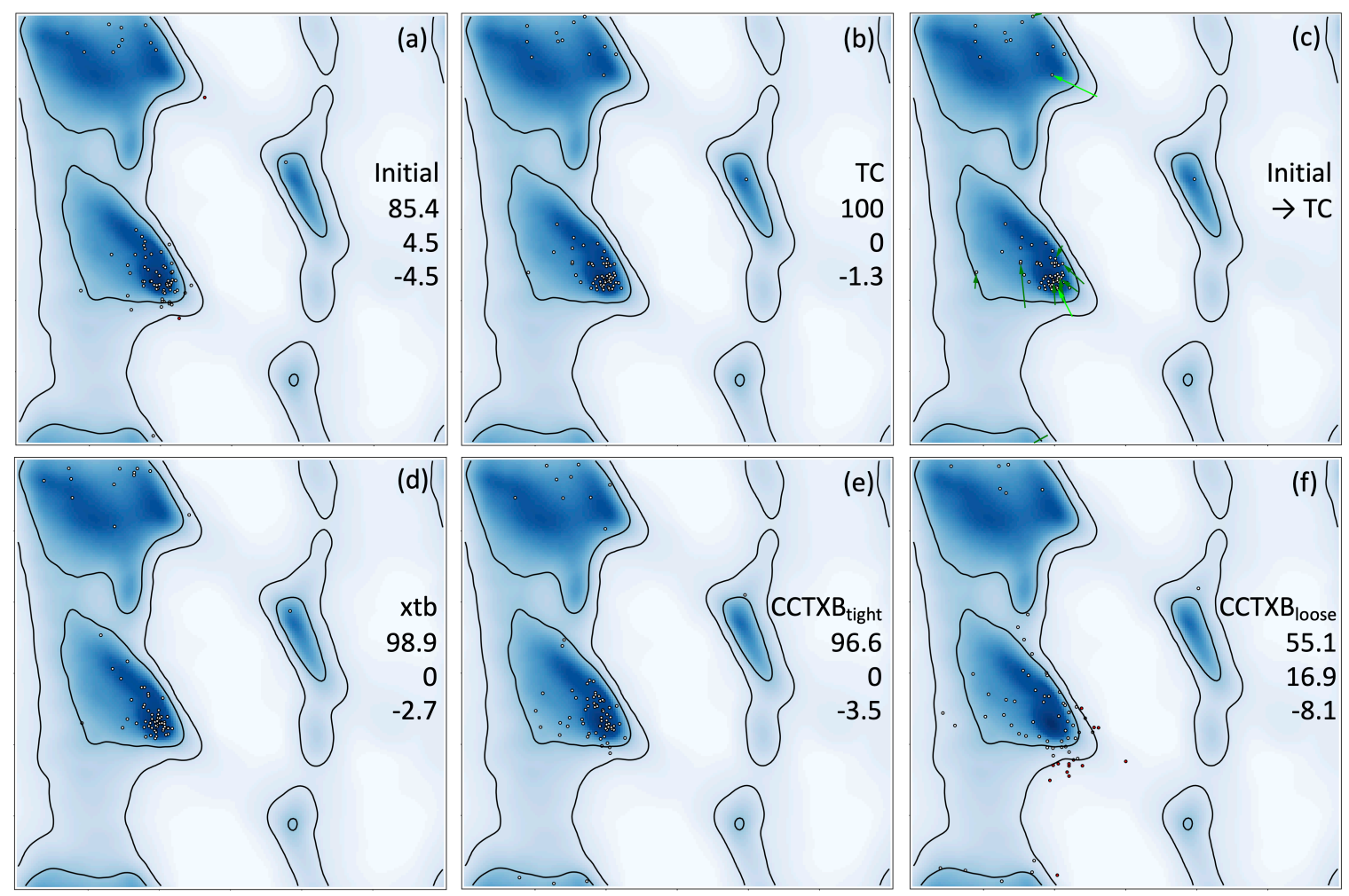

Figure 1. Ramachandran plots for 3j63: (a) initial; after quantum refinement using TeraChem (b,c) and xtb (d); and after classic refinement (CCTBX) using tight (e) and loose (f) geometry restraints. Columns of three numbers show percentage of residues in favored and disallowed regions of the plot, and the Rama-Z score. The Green and light-green arrows on the panel (c) show residues moved from allowed and disallowed regions to favored region after TC refinement. 

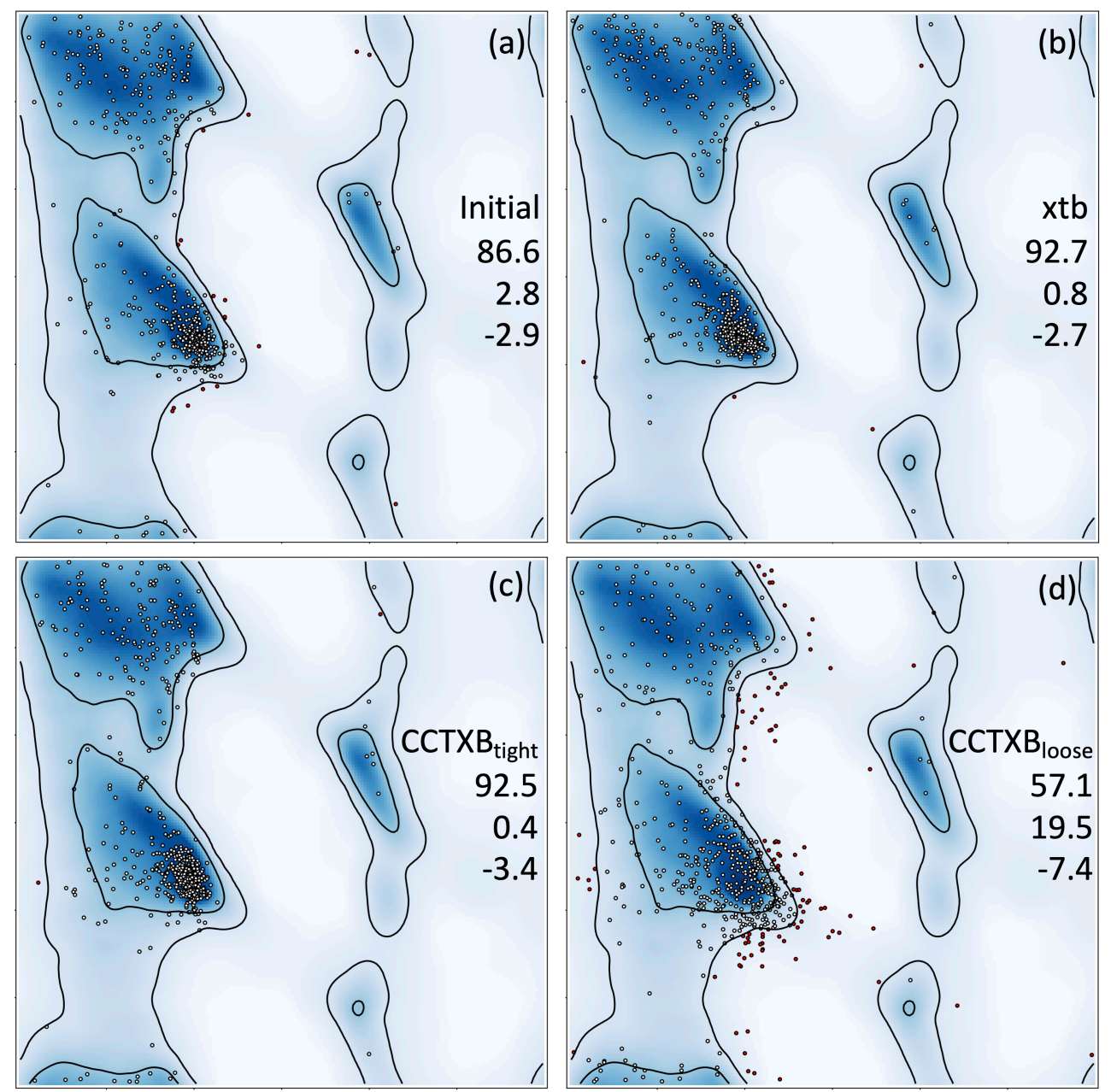

Figure 2. Each Ramachandran plot shows residues accumulated from all three models considered in this work (3j63, $5 \mathrm{fn} 5$ and $3 \mathrm{a} 5 \mathrm{x}$ ): (a) initial, (b) after quantum refinement (xtb) and after classic refinement (CCTBX) using tight (c) and loose (d) geometry restraints. Columns of three numbers show percentage of residues in favored and disallowed regions of the plot, and the Rama-Z score. 

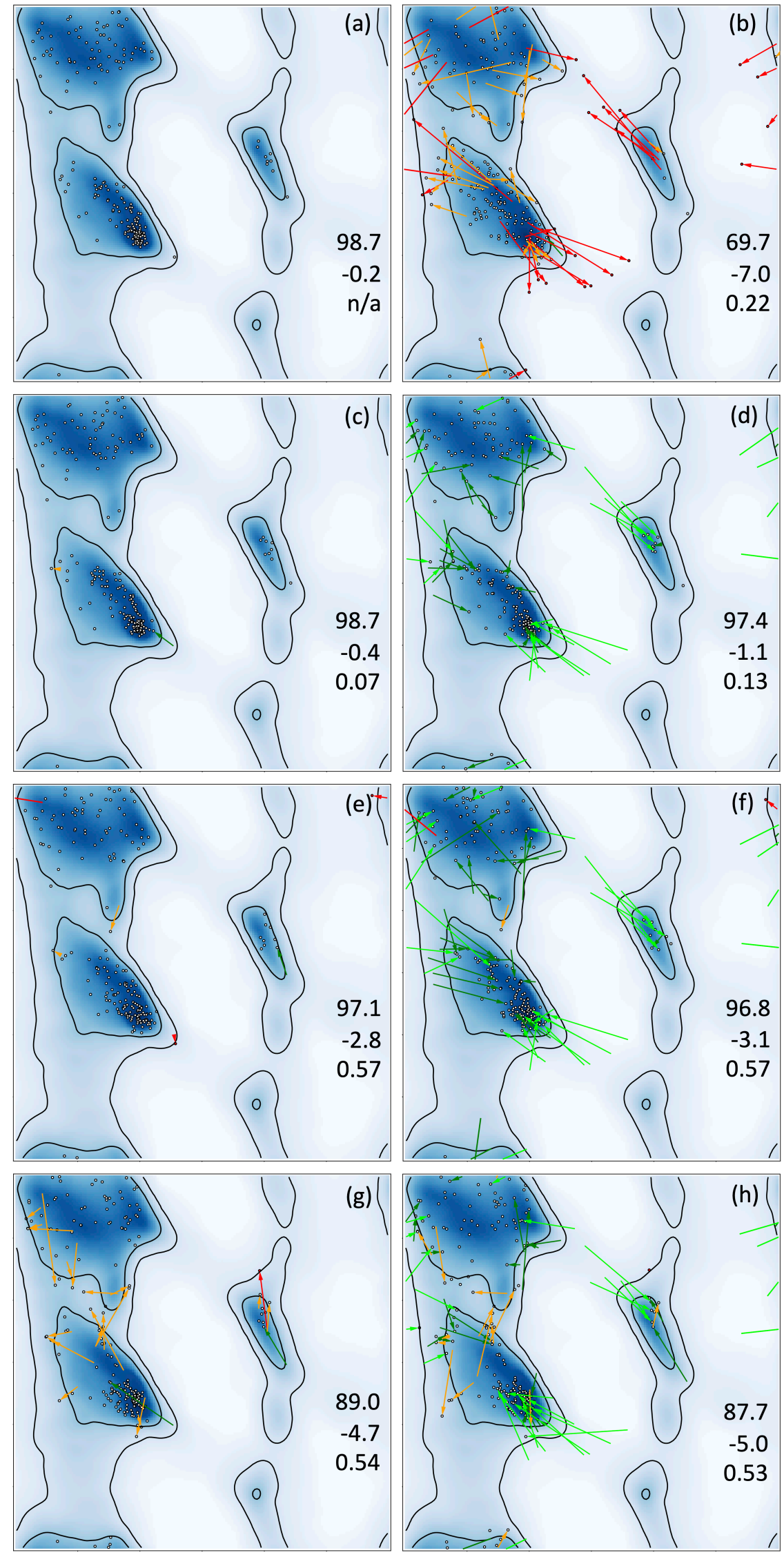
Figure 3. Ramachandran plots for 1us0: (a) original model, (b) perturbed model, (c, d) original and perturbed models, respectively, after QM optimization with TeraChem, (e, f) original and perturbed models, respectively, after QM optimization with $\mathrm{xtb}$ and $(\mathrm{g}, \mathrm{h})$ original and perturbed model, respectively, after classic optimization. Red and orange arrows show residues moved to disallowed and allowed regions out of favored region as result of perturbation. Green and lightgreen arrows show residues moved from allowed and disallowed regions to favored region. Columns of three numbers in each plot show percentage of residues in the favored region of the plot, Rama-Z score and RMSD (in $\AA$ ) to the original (a) model. $(\mathrm{b}, \mathrm{c}, \mathrm{d}, \mathrm{e}, \mathrm{f}, \mathrm{g}, \mathrm{h})$ plots were created using phenix.comparama. 